\title{
On the Relation between the Comprehension Ability and the Neocortex Verbal Areas
}

\author{
André Michaud* \\ President of SRP Inc, Canada
}

\begin{abstract}
General description of the human neocortex verbal areas and exploration of the manner in which the synaptic neurolinguistic structure that develops in these areas after birth establishes our comprehension ability. Description of the manner in which the neurolinguistic subjective model of reality that develops in these areas can be made to evolve towards objective representation.
\end{abstract}

Keywords: Pavlov; Chauchard; Hebb; Neocortex; Verbal areas; Conceptual thinking; Comprehension process; Subjective model; Objective model

\section{Introduction}

A common belief is that the thinking process is something abstract that will forever escape our understanding. Nothing is further from the truth. In fact, the first groundbreaking understanding of the human thinking process was accomplished by neurophysiologist Ivan P. Pavlov towards the end of the 1920s, as he became aware of the direct relationship between thinking and language $[1,2]$.

Unfortunately, this major conclusion of Pavlov was barely noticed in the scientific community, because to this author's knowledge, his related 1932 essay "Versuch einer physiologischen Interpretation der Symptomatologie der Hysterie" ([2], p. 265) has not yet been translated to English, and was published in German only in 1998.

Some scientists in France paid attention however, and Paul Chauchard, renowned neurophysiologist, Director of research at "École des hautes études" in the 1940's and 50's, continued Pavlov's work in collaboration with other French researchers until they discovered the direct relationship between the level of language mastery, and the level of ease and correctness with which individuals can comprehend issues, which corresponds in fact, to the understanding level reached by an individual, that is, "intelligence" [1]. It turns out that the degree of awakening of intelligence, that is, of the comprehension ability, is a direct function of the degree of language mastery $[3,4]$.

Metaphorically speaking, the language mastery level can be compared to the resolution level in cameras. The best satellite cameras in 1997 had resolutions that did not allow identifying objects smaller than an automobile at the surface of the earth, because objects relatively smaller than a single pixel of these cameras' resolution were de facto invisible. More recent satellite cameras can identify smaller objects due to higher resolution, that is, by making use of smaller pixels.

Similarly, Chauchard and these other researchers found that we are unable to understand the subtle nuances of situations, or describe objects any more clearly than the extent of vocabulary and general language mastery that we possess.

For example, when looking at a car engine, a person with no notion of mechanics will think of it as simply the "engine" and when asked to describe it, will not typically be able to give much details about it other than that it is what propels the car, and describe in general terms its various outside shapes and outstanding features.

A mechanically savvy individual on the other hand will be able to say from a single glance, for example, that it is a four cylinder front wheel drive engine with turbo compressor and built-in transverse transmission, or that it is an old style direct injection V-8 engine for rear wheel drive.

This second individual may also have a fairly good idea of the inner working of the various components and be able to coherently describe these inner components to some extent, while the first individual may have nothing come to mind when trying to fathom what could be inside the engine block.

Finally, an actual mechanics can dismantle the engine, describe in detail every part as he goes, explain their function, and reassemble with ease the engine back to working status.

Obviously, the descriptions of all three individuals are correct, but to varying degrees of precision. It can easily be understood that even if the first individual does not have the term "turbo compressor" in his vocabulary, he can nevertheless see the turbo compressor. But having no knowledge about it, nor any word to name it, he will hardly be able to even identify which part of his concept of "engine" it relates to and even realize that it is a component separate from the engine. He will consequently be unable to even "think" about it.

The difference in understanding ability between the three individuals on the issue of engine is strictly one of extent of vocabulary and related information, going from bare minimum understanding for the first individual to completely objective understanding for the third individual. Obviously, this relation between vocabulary and understanding ability applies to all issues, implying that the general understanding ability increases in the same proportion as individuals general knowledge base, the acquisition of which is what causes the concomitant vocabulary increase.

It can then be understood that to "think" about something, "knowledge" and "words" are required. So the conclusion is that the

${ }^{*}$ Corresponding author: Michaud A, President of SRP Inc, Canada, E-mail: srp2@srpinc.org

Received December 22, 2016; Accepted January 25, 2017; Published January 30, 2017

Citation: Michaud A (2017) On the Relation between the Comprehension Ability and the Neocortex Verbal Areas. J Biom Biostat 8: 331. doi:10.4172/21556180.1000331

Copyright: @ 2017 Michaud A. This is an open-access article distributed under the terms of the Creative Commons Attribution License, which permits unrestricted use, distribution, and reproduction in any medium, provided the original author and source are credited. 
more" knowledge" we possess about something and the more words we have at our disposal to describe its various "characteristics", the clearer we can "think" about it and consequently describe it. For those "characteristics" that we do not have a specific word for, we can always mentally use a "verbal expression", which amounts to a "verbal definition" that replaces a missing specific word $[3,4]$.

For all intents and purposes, it seems impossible for us to think about events, objects, concepts, etc. any more clearly than the degree to which we master the instrument that we use to think about them, that is, the language that we use. Optimal mastery will then allow optimal ease of expression through strict use of language itself, as when we write, and also through simpler oral language typically associated with complementary non-verbal "body language" when speaking with another person.

\section{Relationship between intelligence and ease of expression}

This discovery highlights the fact that the belief that each individual would be endowed with a fixed and genetically determined degree of intelligence can only be totally false. It is a fact that the ease with which people understand things does vary, sometimes considerably, among individuals, but this condition is neither fixed nor genetically determined.

In reality, it is the end result of the degree of ease in verbal expression that each individual will have developed, in conjunction with the ease with which he or she will accept to reconsider his own conclusions.

This ease in verbal expression and the readiness with which a person will accept to reconsider any of his or her conclusions can increase or decrease during the course of a person's life in relation with the type of intellectual activities that the individual engages in. In other words, the degree of an individual's intelligence can vary, with highs and lows, throughout his or her life.

An individual who constantly struggles to understand situations or to resolve issues of any nature, who reads or speaks a lot about an large array of issues has a tendency to acquire a deeper ability of understanding than one who does not indulge in such activities.

Likewise, a person not previously indulging in any of these activities, but who begins steadily exercising one or more of them, will see his or her general ability to understand increase. If a person stops exercising these activities, he or she will eventually notice a gradual decrease in his or her general understanding ability.

These changes are of a gradual nature, and extend over sufficiently long periods of time for us not to generally become aware of them, just like we are unable to directly become aware of the growing process of a tree. Generally speaking, as with all other activities, the more we exercise appropriate methods of verbal thinking, the more skillful we become in using them. The nervous system is of such nature, that the parts that are exercised the most are strengthened by such frequent use and become easier to use.

According to Pavlov's discoveries ([2], p. 256), the human thinking process is characterized by two distinct aspects, which are the thinking mode by images association ("image" taken here in a very general sense), and the thinking mode by words association $[1,3,4]$. The first mode is the consequence of our direct awareness of the perceptions of our senses and emotions, which amounts to the "knowledge" previously mentioned, and the second mode is the language that we use to describe and understand these perceptions that we become aware of.
In other words, our awareness, or "consciousness of being", whatever this might be, observes the external world in which we live and the internal world of our emotions by means of the image thinking mode (non-verbal thinking), but describes and understands it by means of the language that we use to think about them (verbal thinking).

According to Chauchard's conclusions, the non-verbal thinking mode provides us with a simple way of thinking by images association as when daydreaming for example, as when we automatically avoid obstacles as we walk, or as when we simply react, without really analyzing, to various daily situations, etc.

We presently share this thinking mode by images association with all other mammals, that also possess a neocortex, and some bird species for which this activity is supported by a different brain structure. According to Chauchard's observations, in the case of animals, thinking by images can in no way compare to the vastly superior quality and precision of this process in humans, on account of the much greater complexity of our neocortex.

In addition to this superior ability to use the thinking mode by images association, our neocortex provides us with neurological support for an ability that is available only in a grossly rudimentary form in the more highly evolved animal species [5], which is the actual support of our intelligence, which is the verbal thinking mode, source of our unlimited "comprehension ability", that allows us to abstract and generalize the innumerable signals making up the images that the thinking mode by images provides us with, which is what Pavlov discovered.

\section{The level of intelligence can be controlled}

Given the very direct connection now identified between language and intelligence, it becomes possible to adapt teaching methods to directly influence the language learning process during childhood, so as to enhance children's mastery of language to as high a level as possible, which will in turn enhance their ability to comprehend.

The structure of the verbal areas in both male and female humans of all races being identical, it can also be concluded that any individual of our species can potentially attain the highest levels of intellectual achievement. So, whenever children are referred to as "him" or "he" for simplicity's sake in this text, it should be understood that the term refers with no restriction to all children of all races and of both sexes of our species.

\section{The Human Neocortex}

The human brain is well known to be a highly complex organ, comprised of approximately 100 billion neurons. Only one of its components, however, is of particular interest to us here, which is the outermost layer of the brain, or neocortex. The reason for this particular interest is that this thin external layer of the brain is the seat of memory, conceptual thinking and self awareness of the individual.

Considered from a certain angle, the neocortex could even be viewed as the very essence of the individual, all other brain sub-systems being required only to feed it and provide it with the information originating from the external world, which is required to sustain conceptual thinking, the rest of the body being there only to insure its survival.

We use to consider that we see with our eyes, hear with our ears, feel with our skin, etc. This is true of course at the general level. But at the biological level, the millions upon millions of nerve endings connected 
to each of our senses perceive a continuous flow of as many millions upon millions of separate events, that are detected at the molecular level, and feed them directly to the entry layer of various areas of our neocortex ([6], p. 287), which "processes" them before delivering them in a form that our "conscious mind" can make sense of.

In reality, our neocortex is the physical interface between external physical reality ([7], Section II) and our awareness. All external stimuli coming to our nerve endings are ultimately fed into the entry layer of this 6-layer neural network as a continuous flow, and after having been processed by the intercalary layers, provide all that we can become aware of at the output layer. In a certain way, this output layer of the neocortex is the only "window", or "screen", through which our "conscious mind" can observe external physical reality and the inner "world" of our emotions and conscious thoughts.

Chauchard referred to the neocortex as the "thinking network", a quite fitting name, considering that conceptual thinking seems to be an endless journey among the memories stored within this neural network.

The neocortex is in fact the most extraordinary and complex structure in existence. Only 2 to 3 millimeters thick, its total approximate surface area reaches a surprising $1924 \mathrm{~cm}^{2}$. This thin sheet is made, according to Chauchard [5], of no less than 14 billion neurons of three different types generally organized in 6 layers (about 10 billion, according to Eccles [8]). The number of synaptic links between these cells is practically impossible to estimate with any precision. It generally is agreed that neurons can be linked to as many as 10,000 to 100,000 other neurons, which allows considering total numbers of synaptic connections in the tens of thousands of billions within the neocortex.

To give an idea of the disproportion between the number of neurons making up a neural network and the number of links that can possibly be established between them, given that a neuron can potentially establish links with all neurons of other layers, a group of as few as 300 neurons can quite realistically establish as many as 20,000 links within the group.

Such an unfathomable number of links within the neocortex takes on a particular significance when we consider, as did Chauchard, that it is not the number of neurons that counts to explain Man's superiority over other species, but the density of the interconnected network ([8], p. 58); and that associative memory rests on the synaptic interconnection network and not on the neurons proper, as Hebb also concluded in 1949 [7,8] ([6], p. 640) ([9], p. 146). According to him, the processes of learning and memorizing involve changes in the intensity with which the electrochemical nervous signals are transmitted through individual synapses. Consequently, the astronomical number of synaptic links present within the neocortex of every human being hints at a phenomenal processing capacity.

The expression "processing capacity" immediately brings computers to mind, which leads us to attempt comparing them to the neocortex. But let us not be deceived! Contrary to generally accepted beliefs, particularly in the computing community, the actual processing power of even the most powerful existing linear or parallel super computer is quite insignificant when compared to that of the neocortex.

As a matter of fact, linear processing computers are unable of processing more than one instruction or data item at a time (a little more with processors in parallel banks), which leaves them forever behind neural networks, very far behind the neocortex, which, like all neural networks, can simultaneously examine as many data items as there are neurons in the entry layer of the neocortex area concerned by each of our sense, and instantly offer in real time at the output layer the coherent "images" perceived in the entry signals set.

Even the best neural network simulation programs, by necessity designed to operate on linear or parallel computers, are subject to this constraint of having only one instruction processed at a time, which degrades their performance to the point of giving only a pale idea of the real processing capacity of true neural networks.

The reader can become aware in a very simple manner of the processing capacity of his own visual cortex, an area of the neocortex located at the back of the brain (Figure 1), Vi), made up of about 400 million neurons ([8], p. 263). Simply looking around from side to side for a few seconds shows how easily our visual cortex identifies visual coherences in real time, coherences that we become aware of as they are "displayed", so to speak, at the output layer of the visual area, as the constantly changing visual information is provided at its entry layer, made of about one million ganglion neurons for each eye, to which in excess of 100 million rods and cones receptor cells of each of our retinae are connected.

Each ganglion cell receives the pre-processed signals from about 100 cone and rods cells that are constantly being hit by the incoming light photons, defining a wide range of signal intensity for each ganglion cell, which hints at way higher effective resolution than the approximate one million entry ganglion cell per eye suggests. Strangely enough, this entry layer of the visual area is located rather far from the rest of the neocortex, that is, directly in each eye retina.

As we slowly turn our head, looking around, the detectable signals sent by the retinae cells activate the entry layer of our visual area and the coherences that exist in the entry signals sets are "automatically" made available at the output layer. This is how the visual area output layer, of which our "conscious mind" is aware of, lets us perceive in real time what enters our field of vision. This output layer is available for our awareness (whatever that may be) to consciously observe, either voluntarily or when something attracts our attention, or to ignore, as when we are daydreaming or concentrating on some inner issue, trying to resolve it.

The amount of information provided to the entry layer of the visual cortex by the receptor cells of the retina, which are hit every second by huge quantities of photons coming from the environment is literally fantastic and constantly renewed. We can observe that this does not prevent the network from effortlessly keeping up with the flow, for as many hours as we need every day, during our whole lifetime.

The reader can rest assured that despite the well known slowness of the nervous influx in neurons and of the chemical reactions in the

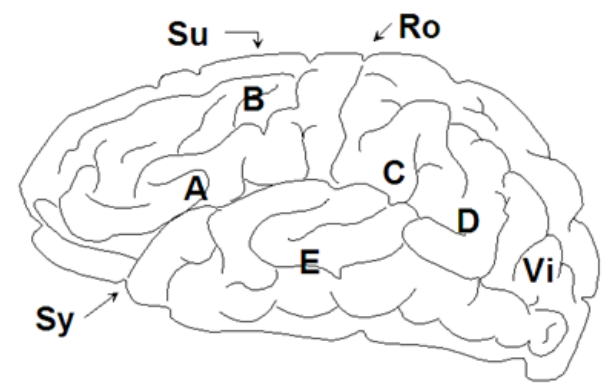

Figure 1: The verbal areas of the neocortex 
synapses, with respect to the phenomenal execution speeds reached by modern linear super computers, that such performance is still totally out of reach of the latter, amounting to simultaneous processing in real time in excess of 100 million pixels for each changing image provided. The secret lies in the simultaneous data elements processing ability of neural networks with respect to the linear processing of conventional computers.

Another and even more striking example of the natural ability of the neocortex neural network to perceive available coherences in sets of elements is the following, that was the object of many conversations on the Internet for a while:

"Aoccdrnig to a rscheearch sdtuy at Cmabrigde Uinervtisy, it deosn't mttaer in waht oredr the ltteers in a wrod are, the olny iprmoetnt tihng is taht the frist and lsat ltteer be in the rghit pclae. The rset can be a total mses and you can sitll raed it wouthit any porbelm. Tihs is bcuseae the huamn mnid deos not raed ervey lteter by istlef, but the wrod as a wlohe."

The fact that the groups of letters are clearly separated by blanks (which informs our neocortex that they "may" be words in a sentence), and that they are clearly delimited by their proper first and last letter (which immediately points the network towards the few most probable solutions for each word), cause the network to automatically consider the most probable coherence for each group of letters in light of the context, that becomes clearer and clearer as decoding of the sentence proceeds.

Besides, we can notice that if any of these groups of letters was proposed without mentioning the others, the information that it possibly is a word in a sentence becomes much less obvious, and it may then even remain totally unintelligible.

Presently, neural networks are not computers, in the generally understood meaning of the word, but correlators. Which means that they process information by concurrently correlating the data elements in whatever data set is provided at the entry layer, which causes whatever coherence or pattern that could be present in the entry data elements set to become available at the output layer. It goes without saying that at least one coherence must be present in the entry set for the network to detect. In fact, neural networks are functionally unable of recognizing anything whatsoever in data elements sets that do not inherently contain some sort of pattern $[10,11]$.

Of course, conceptual thinking and self awareness require much more than just being able to become aware of coherences perceived in the environment by the visual cortex. In fact, it requires the coordinated integration in real time of the whole collection of coherences resulting from the sensory perceptions, emotions and body motions experienced by the individual.

As Chauchard so well formulated with regards to the neocortex output layer and the coherences that it provides:

"it is only of this coordinated set that we are aware. We do not know the detail of all the incoming messages, but only of the global interpretation, which is the only thing that matters. The primary cerebral interpretation tasks are unconscious ([12], p. 59)."

The unconscious "interpretation tasks" that Chauchard mentions, refer of course to the automatic coherence identification process that takes place within the thickness of the 4 inner neocortex layers as the input signals propagate from the entry layer to the output layer.

The understanding of the working of neural networks completely confirms this observation, since by very structure, it is physically impossible for us to become aware of the individual signals reaching the neocortex entry layer from the nerve endings. Only the resulting global coherences that become available at the output layer can possibly draw our attention.

To that effect, the neocortex is subdivided into a mosaic of regions; each assigned a very precise task. Each of these areas possesses the same phenomenal processing power as the visual cortex that we have just become aware of.

Even though the intellectual superiority of our species over any other living species makes no doubt, the reasons for this superiority are not necessarily obvious, and still remain the subject of heated debates.

However, the evolution of species towards complexification gives us an unmistakable telltale at the neurophysiological level at least, of one major reason for that superiority of mankind. It relates to the appearance with the first hominid species (homo habilis, and then homo erectus), of two new areas in one of the hemispheres of the brain, areas that exist in no other species, not even in those closest to us, and that reached their full development, according to our current knowledge base, only with neanderthalians and homo sapiens. They are the Broca area and a large segment of the Wernicke area, which are genetically designed to support language in human beings ([8], p. 122).

As Pavlov observed, this latest push of the evolutionary process has thus modified the genetic code of our species to insure that our neocortex develops these structures that are required to support articulate language ([2], p. 256).

We will now explore the 2 main aspects of the incredible neurolinguistic structure that progressively comes into being within these verbal areas from birth onwards under the pressure of the manner in which language is learned; extraordinary metaphorical virtual multidimensional labyrinth within which our awareness endlessly circulates, intensely "illuminating" each "room" that it visits as our attention focuses, and from each of which we guess more than we clearly perceive, a number of passageways seem to lead away in all directions.

\section{The Function of Each Hemisphere}

As previously mentioned, the appearance and development of the Broca and Wernicke verbal areas, occur in only one of the two hemispheres of the brain. This asymmetric development allows the non-verbal hemisphere to conserve the same general structure as in the species that preceded us.

This hemisphere thus generally retains its usual function of memorizing the whole collection of sensory perceptions, sequences of non verbal events, etc. that support the same type of non verbal thinking which is characteristic of superior species close to us. In our case however, the considerably increased density of the interconnected network with respect to that of other species provides us with an infinitely superior quality of non verbal thinking with respect to these other species ([4], p. 119).

The cephalization coefficient established by Dubois has been found to be four times higher in the case of human beings with respect to anthropoids, and it was also established that 33 genetically programmed cellular bipartitions are required to construct a human brain compared to only 31 for anthropoids ([12], p. 92).

The awareness that we have of our sensory perceptions eventually 
induces a cerebral structuring of specific sets of imprints which represent and replace in our awareness the objects and events considered, and that become the thought or idea that we have of these objects.

"To think, is to associate cerebral images (giving here to the word "image" a general meaning extending beyond mere sight) that education creates within us from the sensory messages, that can be conjured up through imagination, and that have thus become an interior code, an autonomous manner in which our brain can be used ([4], p. 121)."

\section{Paul Chauchard, 1960}

It is this form of non verbal thinking that Pavlov named the "first system of signalization". Superior animals possess only this form of thinking, but at a much more elementary level. The verbal hemisphere on its parts, takes care in human beings of organizing and generalizing the non verbal "images" of the opposite hemisphere.

"It was Pavlov who showed that language was a consequence of the human cerebral complexity and that it objectified the superiority and specificity of the human brain with respect to animal brains. He perceived language as a special type of conditioned reflexes, a second system of signalization. The first being that of gnosis and praxis of direct thinking by images.

To each image is substituted through education its verbal denomination. Since they name everything, instead of associating images, human beings can directly associate the corresponding names, a system more adequate to fully extend the abstraction capabilities of the human brain ([4], p. 122)."

\section{Paul Chauchard, 1960}

\section{Functional asymmetry}

Contrary to the state of asymmetry that characterizes the hemispheres in human beings, Hamilton's studies have clearly demonstrated an absolute chiral symmetry of the cerebral hemispheres of rhesus monkeys. That is to say that no unilateral memorization, nor any difference in ability, neither in performance nor in ease of training, could be noted between both hemispheres, which led him to conclude in 1977 that the weight of all experimentally gathered data confirmed the theory that there is no specialization of one hemisphere in rhesus monkeys [13-15].

No other exhaustive study was reported as of 1999 for other species of apes or monkeys, but the bulk of available information indicates that they are generally ambidextrous and that no manual preference was noted.

From all indications then, we would be the only species in existence to possess such an asymmetry of the hemispheres, a uniqueness also confirmed by the fact that no other species possesses an articulate conceptual language. All aspects of this asymmetry are clearly highlighted by Eccles in his magnificent book "Évolution du cerveau et création de la conscience" ([8], p. 263), which is a priceless source of references about the bulk of the research that was conducted regarding the various aspects of the neurolinguistic evolution of the human brain.

Hemispheric asymmetry is mainly characterized by the localization in the left parietal, temporal and frontal lobes (the right lobes for about half of the left handed population, which constitutes about $10 \%$ of the whole population) of wide areas specialized in the production and comprehension of language, mainly the Wernicke and Broca areas.

Although the corresponding mirror areas of the opposite non verbal hemisphere seem to play no functional role in speech production, an important level of activity has been observed to occur in parallel with this production, mainly in the mirror Wernicke area.

This parallel functioning of the hemispheres is perfectly explained by Goldman and Nauta's extraordinary discovery in 1977 [16], showing that the human neocortex subdivides into a mosaic of modules practically isolated from each other, which would constitute the basic elements of the functional structure of the neocortex. Each of these modules is made up of a limited number of intimately interconnected neurons, only a few axons of which would project to neighboring modules, but with the bulk of the axons going through the corpus callosum to generally (but not always) connect to a similar module located symmetrically in the opposite hemisphere ([8], p. 269).

Levy noted in 1974 that each side of the brain seemed to busy themselves at executing cognitive activities that were logically incompatible with each other but complementary. He concluded that while the right hemisphere executes a synthesis in space, the left hemisphere was doing an analysis over a time period. He also concluded that the right hemisphere notices the visual similarities without reference to conceptual similarities while the left hemisphere did the opposite. He also came to the same conclusion as Pavlov and Chauchard, to the effect that the right hemisphere codes sensory perceptions in terms of images, while the other code them in terms of verbal descriptions ([17], pp. 121-183).

This lateralization and specialization of the verbal areas are of genetic origin. The formation of the language areas in the brain is initiated even before birth ([8], p. 118), and although both hemispheres are actors in initiating the elaboration of language [18], the left hemisphere (the right one, in $5 \%$ of the population) acquires little by little the preponderance for language which is favored by its genetic predisposition. This displacement process usually ends during the fourth or fifth year of childhood [19].

The reason why these regions possess the faculty to learn all languages is due to the fact that the neurons of these areas are only weakly interconnected at birth, and the bulk of the verbal synaptic network of each individual, structures itself specifically according to the particularities of the language or languages being learned and under the pressure of this learning, in the period during which most of this synaptic construction naturally occurs for all individuals, that is, during the optimally favorable period between birth and the age of approximately 7 , age at which complete myelinization of these areas is genetically triggered, and also during adolescence ([4], p. 41).

It is observed that even during their first months of life, children continuously exercise their phonation organs and thus learn to use this organ that requires the most complex of motor coordination sequences ([8], p. 101). Even if hemispheric asymmetry and predisposition to learn languages are of genetic origin, the full development of the areas concerned depends entirely on environmental circumstances ([20,21], p. 78 ). It was conclusively demonstrated that if the language areas are not used before puberty, they lose their capacity to learn [21] and ([8], p. 112).

As early as 1920, Flechsig discovered that the Broca and Wernicke areas were the last to completely myelinize, this myelinization occurring only after the full dendritic development of these areas, an event that coincides with the end of childhood [22] ([8], p. 121).

It was also confirmed by Chauchard that the human brain is completely developed, that is, presents a completed neural network, 
as children reach approximately 7 years of age ([4], p. 45), which means, given that the development of the verbal areas depends on environmental conditions, that to obtain an optimal development of these areas, it is mandatory that all aspects of verbal expression, auditory recognition, reading and writing be mastered at least to a level of ease before this critical age, because all indications are that if sufficient mastery of any of these aspects is not completed during childhood, they become more difficult to acquire later ([4], p. 52).

This is why children who are lucky enough to have been taught to read before this myelinization deadline are more likely to develop a taste for reading and eventually become aware that this gives them access to all of the accumulated knowledge of humanity.

In view of the confirmed observations of Lenneberg [21] regarding the consequences of neglect in using the verbal areas under construction, given that the verbal areas are barely interconnected at birth despite the presence of their full complement of neurons, and that the full synaptic interconnecting network will develop from birth on through childhood, there is no doubt that their organization is strongly influenced by the manner in which the various aspects of language are acquired.

It is consequently certain that any neglect in providing the various verbal trainings to the level of ease of use during the active network construction period of childhood will have a marked impact on the density of the network that will be directly associated with these aspects of language, a density that Chauchard directly associates to the level of intelligence that would eventually be attainable by the individual.

"Once the normal age for the development of the verbal areas is passed, such training will become difficult.

The fundamental law of cerebral development, that is, the possibility of possessing later a completely normal brain, endowed with all of the human aptitudes, requires that cerebral maturation always takes place in an environment, not only physical, but cultural and affective, that will favor it. Nothing can be done too soon, but soon, it is too late ([4], p. 52)."

\section{Paul Chauchard, 1960}

\section{Structure of the verbal hemisphere}

Let us now examine the general organization of the verbal areas.

The location in the neocortex where the sequences of motion that must be executed by the phonation organs to pronounce each word are stored is the Broca area (Figure 1), A) (also identified as the Brodmann zones 44 and 45, 1909). It constitutes a wide area of the left prefrontal lobe, located just in front of the motor center of vocalization, right above the Lateral Cerebral Fissure (Figure 1), Sy), and that Chauchard also names the center of verbal articulation ([8], p. 110) ([4], p. 110).

This localization is an established fact, because unfortunate accidents that cause the destruction of this area of the brain systematically cause the victims to become practically unable to speak, even though their ability to coherently understand, read and write words is not affected. Chauchard locates the center of writing praxis a little higher (Figure 1), B), somewhere in Brodmann zone 4, facing the motor center of the upper limbs ([4], p. 110) ([5], p. 48).

The area where the synaptic imprints of each word are created in the left temporal lobe is also well known. It is the Wernicke area (Bodmann zones 39 and 40, as well as parts of zones 21, 22 and 37, 1909). Chauchard subdivides the Wernicke area in three sub-areas: 1- the sensory center of language (Figure 1), C) (Brodmann zone 40), 2- the reading center (Figure 1), D) (Brodmann zone 39) and 3- the words auditory center (Figure 1), E) (parts of Brodmann zones 21, 22 and 37). The verbal areas are also summarily described in reference [6].

The synaptic networks of these areas grow and interconnect during infancy as a function of the intensity with which the child practices the various activities that they support. A satisfactory level of structuring still remains possible afterwards, but with more difficulty, mainly by means of extra training of the synaptic structure that was established during the infancy active construction period.

Each verbal imprint in the Wernicke area is connected at the synaptic level 1) to the sequence of phonation motions stored in the Broca area that must be executed to pronounce the corresponding word; 2) to the sequence of motions stored in the writing center that are required to write the word; 3 ) to its imprint in the reading center; 4) to the imprint located in the auditory center; 5) and finally, to all of the various aspects of the recollections that have been associated with it over time, which are stored as non verbal image imprints in the mirror Wernicke area in the opposite hemisphere.

For deaf persons who learn sign language or lip reading, it is of course the center of vision which is connected in "input", so to speak, to the verbal areas to allow recognized signed words to be routed to that center. For these same individuals, the motor centers that control the arms, hands and fingers are connected in "output" to the verbal areas if they learn sign language. For persons that hear normally and also for certain deaf persons, the motor centers controlling the movements of the mouth, tongue, vocal cords, etc are also connected in "output" to the center of verbal thought.

For blind persons who learn Braille, the centers controlling the sense of touch in the hands are connected in "input" to the verbal areas to allow words recognized by touch to be routed to these areas.

The localization of the synaptic imprints corresponding to each word in the Wernicke area is quite certain again here, because accidental destruction in this area always rendered the subject unable of comprehending what they read or what is being said to them, while still remaining capable of speaking (if the Broca area was not affected), but producing only absurd incoherent phrases, totally devoid of meaning ([8], p. 110).

The mere fact that the whole collection of synaptic word-imprints that are created come to be separately established, in the Wernicke area, as each word is learned, seems insufficient to explain how the whole set of words could associate to constitute a coherent interconnected global structure that would allow us to think by means of combining these verbal imprints, that is, by building coherent phrases.

It is very interesting in this regard to observe that there exists a third area dedicated to language. This special area is located at the very top of Brodmann zone 6, and is named the supplementary motor area (Figure 1), $\mathrm{Su}$ ). What is special with this area is that it has been experimentally demonstrated to be the first area to activate when an individual is about to speak ([8], p. 113).

Consequently, it seems altogether possible that this area could be the summit of the structure, so to speak, the place where a synaptic link is established with each of the verbal imprints of the Wernicke area, that would allow associating at will those words that momentarily attract the attention of the person, either to express them verbally or in writing, or to simply think conceptually, without necessarily activating the phonation praxis of the Broca area. It is therefore not impossible 
that the apex of the whole neurolinguistic structure that we will explore further on could reside exactly here.

On the other hand, the same action initiating mechanism seems quite capable of routing links to the various aspects of the recollections that the individual is attempting to correlate towards the frontal lobes, which are known to enter into action when an individual deeply cogitates.

Eccles reveals that it was experimentally confirmed that the seat of "self awareness" is located in the hemisphere that contains the language centers. Consequently, the leap of imagination is very small to conclude that even "self awareness" could be an outcome of the very existence of the neurolinguistic synaptic structure that develops under the impulsion of language learning. Chauchard explores in depth the various aspects of self awareness with respect to language in his book "Le langage et la pensée" [5].

\section{Artificial Neural Networks}

Before examining how the first learning experiences of children lead to the development of the neurolinguistic synaptic structure that supports conceptual thinking, let us momentarily go back to Hebb's explorations regarding multilayer artificial neural networks, because he observed that these artificial networks experience the same difficulties in resolving problems that we meet ourselves. In their case, however, the difficulty level becomes extreme [11].

A thoroughly documented example of the difficulties involved in teaching neural networks is given in Chapter 17 of Anderson's quite elaborate "Introduction to Neural Networks" [9], where a detailed attempt at teaching arithmetic operations to a neural network is documented. Note that in this reference, "image" representation is named "analog" representation, while the corresponding "word" representation is named "symbolic" representation.

The parallel that can be made between artificial neural networks and the human neocortex, or "the thinking network", to paraphrase Chauchard, is important to consider, given that learning by human beings and by artificial neural networks is done by training in both cases. The conclusions that can be drawn from the way artificial neural networks function in this regard will allow us to more readily understand the functioning of our own thinking processes.

Artificial multilayer neural network computers are not very well known to the public, as well as within the scientific community in general, where there is often confusion between real artificial multilayer neural networks and the neural networks simulating programs that can be run on conventional linear computers. There are many reasons for this state of fact. First, they are very difficult to manufacture, but their most arresting feature is the fact that, contrary to single or double layer neural networks, they cannot be programmed in any conventional manner.

Consequently, they are not very widely used and the more complex models are still considered laboratory curiosities despite peaks of sporadic research spanning the past 60 years. They are attempts at building computers that could resolve problems in the same manner as we humans.

As previously mentioned, it is impossible to program them like conventional computers. This is what renders them so impractical. Hundreds of hours of training may be required to train them to solve some types of problems. The type of difficulties encountered by the trainers are of the same nature as the difficulties met when teaching children, but increased to the limit [9].
What allows us to identify a coherent solution to a given problem is the use of logic. Even when considering a set of elements in which more than one subset can be identified, we make use of logic to select the best suited subset to be used in context. This level of logic is driven by simple preference, meaning that we simply "feel" that within the reference frame established by the context, a given subset is better suited for the purpose. This is where artificial networks are irretrievably handicapped with respect to us.

Because they are not alive, they do not have feelings, and despite the fact that they easily perceive coherences in data sets just like us, they are unable to determine by themselves which coherence would be the most appropriate in a set when more than on is possible. The notion of "preference" that depends on whether a given subset appears more "satisfying" or "pleasing" in context, is a notion that up to now has been impossible to program into artificial neural networks.

To the disappointed surprise of the first researchers, it was discovered that artificial multilayer neural nets are totally incapable of even the most rudimentary logical reasoning. This is what makes it impossible for them to find answers to problems that we have not already understood and resolved ourselves, before they can be trained to recognize these answers.

They must mandatory be guided "by hand", so to speak, to learn to chose the preferred coherence, otherwise they tend to deliver the first coherence that they perceive, whether it is the best or not, appropriate or not.

What makes it possible to use them despite this handicap, is that when data is provided in input, a coherently selected data subset, however inappropriate it may be, is always provided in output, and that the network can be taught by forced guidance and repetition to eventually always choose the type of subset that it is repeatedly taught to select, thanks to a major discovery made by Donald Hebb about how preferred synaptic pathways are established by being strengthened due to persistent repeated use in the neocortex [10] and ([6], p. 640).

To train a multilayer neural network, the "trainer" must proceed in a very peculiar manner. First, he will provide it with a set of data elements that must be examined and he will analyze the result obtained at the output layer. Various techniques have been elaborated to direct neural networks towards proper solutions, all of which involving gradually adjusting the data provided in input and resubmitting it in a repeated manner to the network [9].

Eventually, there comes a point when the network under training always gives the correct answer. This network is now ready to be used to solve problems of this type that we want it to work on, and will give a pertinent answer in each case in a satisfactory manner.

\section{Language Acquisition in Early Childhood}

Let us now summarize what research on artificial multilayer neural networks has taught us.

- When sets of elements are provided at the input layer, if any coherence or similarity of any sort exists between elements in the set, the subset concerned will be provided at the output layer.

- If many sets of coherences are possible in a given input data set, only one set of coherences at a time can be provided at the output layer.

- Coherences can be selected with respect to more than one criterion. 
- The process of selecting the most appropriate sets of coherences to resolve complex issues must mandatorily be guided by "something".

We obviously are confronted with the same issues with the human neocortex, since we are dealing here with the fundamental characteristics of all neural networks.

It was observed that in the case of young children, their emotions will be this first "guide" in this regard, which will fill the same function for the child that the "trainer" fills for artificial neural networks. This training begins at a very early stage for every child. Their immediate physical needs, like hunger, discomfort, pain, pleasure being the reference frame within which their "preferences" are established in context.

Chauchard very clearly locates the source of the individual's emotions in the hypothalamus ([20], p. 62). It is in this part of the brain, which he identifies as being the seat of what he names "bioawareness", that the whole collection of biological signals coming from other parts the body are integrated and then provided at the input layer of the neocortex as feelings of well being or discomfort. What is detected as properly filling organic needs is interpreted as pleasing to various degrees of intensity at the output layer of the neocortex, and what is detected as being inappropriate or dangerous is perceived as unpleasantness to various degrees of intensity.

"There is here a particularly well developed aspect of bio-consciousness which will show its importance by the fact that the neocortex draws from it critical information required to reach true consciousness, and the means to cause it to grind into existential and organic reality. If we are not an object observable with cold reason, but rather a living and sentient reality, we owe it first and foremost to this hypothalamic integration ([20], p. 63)."

\section{Paul Chauchard, 1958}

All impressions of the senses that children feel are colored by these pleasant/unpleasant impressions originating in the hypothalamus, that are fed to the input layer of his neocortex and stored in the form of recollections, to be used for comparison with any other piece of information.

The impressions of the senses and the internal signals from his physiological needs, bring to his attention his first "pieces of information": sensations of hunger, to be wet, to be uncomfortable for having been in the same position for too long, or to be in a position that he perceives as uncomfortable, or to hear a noise that he is afraid of, etc.

When children don't feel good, for whatever reason, their emotions come into play and they naturally react by crying or screaming their distress. Over time, sequences of events that are repeated often enough end up attracting their attention and one day, the "light" turns on for the first time, so to speak. They suddenly understand that it was their crying and screaming that was causing "someone" to come and do what was needed for them to feel good again. They have just made their first connection between cause and effect!

All parents have noticed at some point that their child began to occasionally cry or scream, apparently voluntarily, since they could identify no cause other than that it appeared to merely be to see if someone would come pay attention. The first logical behavioral patterns make their appearance. From this point on, progress will be constant.

Of course, it is imperative that the parents' reactions and behavior with respect to the child's solicitations be coherent and logical, or else the child will remain confused and his development towards serenity and self control can only be hampered.

As days and weeks go by, the child becomes more and more experienced in evaluating new information and drawing his own conclusions. Little by little, the child discovers how to react to situations so as to obtain the results which are the most satisfying. His natural logic gradually fine tunes until it becomes very reliable. This refinement is induced in the same manner for all children. This is why this natural logic is named "common" sense, since it is a common reference for everyone ([7], Chapter "What is Logic").

We have just put our finger on the reason why artificial neural networks are incapable of learning to evaluate. Since they are not alive, they can neither feel good nor bad, and therefore do not have at their disposal the reference frame required to guide on their own the process of information evaluation. Consequently, they are unable of "taking the first step" which consists in becoming aware that certain conclusions are more "advantageous" with respect to "needs to be fulfilled", contrary to living neural networks whose very survival depends on the well being of the body to which they belong.

As the child grows, he will understand more and more clearly how to draw the most out of every situation with respect to his environment and other individuals. He naturally engages in activities that he prefers, because these situations give him the most satisfaction.

He will use his newly acquired and still somewhat rudimentary natural logic, that is, his increasing ability to evaluate information "with respect to a goal to be reached", to turn situations to his best advantage, inasmuch as he can gain a measure of control over them.

This self training, which results in the acquisition of natural logic, thus precedes speech acquisition. In fact, it is precisely this training to perceive relationships more and more easily between cause and effect that will be the spark that will ignite the glorious verbal explosion that will soon overwhelm his neocortex, and structure it in a manner that will allow coherent thinking.

\section{The First Significant Words}

During their very first months after birth, children continuously exercise their phonation organs, and soon, try imitating the sounds that they hear ([8], p. 101).

We will not dwell on the period during which the child will articulate by imitating sounds or words without understanding them. At some point, children will pronounce their first significant word: "daddy", "mommy", "apple", etc., the full meaning of which having become totally clear to them. At this point in their evolution, children have finally understood that certain sounds, produced by a person taking care of their needs, are always associated with specific objects.

From this moment on, progress will be rapid. However, children often need confirmation. The maelstrom of new non verbal impressions that are constantly coming in, and the speed with which the synaptic connections are being established between the imprints established by these impressions and their ever widening collection of meaningful words, tend to keep them somewhat uncertain of the meanings they have already associated to their established words pool.

They will often repeat certain words, somewhat interrogatively, to obtain confirmation from their parents. Any new object similar to an already named and familiar object will raise questions or interrogative 
attitudes on their part, until they become certain again that they can name it with the same word.

Of course, they will inquire about the name of any new object that attracts his attention.

\section{The Emergence of the Generalization Ability}

Progressively, the whole collection of children's non verbal impressions becomes coherently structured as the progressively more elaborate vocabulary they use to name their various aspects causes these "objects" to be regrouped in various categories. This "ability to generalize", which is thoroughly analyzed in separate references $[13,23]$, and which is the foundation of conceptual thinking, gradually begins to operate, and the child begins to draw and formulate his first indirectconclusions, to the amused surprise of his parents. Often, his first conclusions will be completely off the mark, but the process is now in operation.

It is this generalization ability, that allows children to begin understanding things in the same manner as adults. Since the child has much less information than adults at his disposal, his conclusions, although always logical, can obviously be made only from the very limited pool of knowledge that he is aware of.

If some of a child's conclusions are false from our perspective or seem disconnected from reality, it can only be because his information is incomplete or even false about these issues. It is our responsibility to inform him correctly.

The existence of this freshly matured generalization ability is due exclusively to the existence of the network of synaptic interconnections developing in his brain, that progressively associates the non-verbal synaptic imprints which are the memories (or "images") of the objects or events that he remembers, and the words that he now uses to speak and think about them.

This ability will eventually allow him to understand by himself that an animal that he sees for the first time (a new race of dog, for example) is a dog, because in the past, he would have come to understand and correctly identify the set of characteristics that only dogs possess. In case of doubt, he will ask.

The more he progresses, the more his vocabulary increases and the easier it becomes for him to understand the new impressions coming from the outside world that he becomes aware of, because these new impressions match more and more often impressions that he was already aware of and which he has already named and discussed or simply thought about.

Little by little, he will discover that house pets and people that he "likes" tend to stay longer with him when he is "nice" with them. He will then begin to occasionally include these "loved" beings into his "inner circle", so to speak, meaning that he will experiment with situations in which his conclusions and his actions will also favor other beings besides himself, which are his first steps towards altruistic behavior.

His ability to evaluate situations in order to reach a specific goal becomes more and more refined, and within the restricted frame within which he exercises it, that is, in order to favor himself and the few beings that he likes to be with, this ability becomes more and more reliable, through repetition and correction of the method, as situations warrant.

This type of "experiences" develops therefore his skill at obtaining "good results" within reference frames that include only himself, or himself plus a few beings that are close to him.
Another type of activity, which is natural for all children, is play. Games of all sorts play a determining role in the development of the logical reasoning ability of young children.

All of these games, the objects and circumstances of which can be vary infinitely, supply him with repeated contacts with all sorts of problems that need to be resolved, and in particular, with situations requiring solutions which he is not a part of, such as puzzles, which allows him to begin dealing with abstract issues.

Imaginary situations of which he becomes or not an actor, also involving imaginary characters that he never sees, but which he must take into account, like the Tooth Fairy for example, help train the child in satisfactorily resolving situations that are more and more complex, in an ever widening variety of reference frames.

However, although the growing child is becoming master of the logical reasoning process proper, he will not learn by himself that there is need to verify whether all elements upon which he bases his reasonings are valid. He will not learn by himself either to clearly identify the reference frames within which the reasoning should occur, nor will he learn to clearly define the goal to be reached within such a reference frame, wich can cause him to make erroneous choices even when possessing the correct knowledge.

These other aspects of the formal logic process, that we will examine further on, must be taught to him. Meaning that it is necessary to directly teach him that he must verify the validity of elements upon which he bases his logical reasonings, and to clearly define the reference frames within which he wishes to "analyze" a given situations before arresting his choice of possible solution.

One of the most important aspects of the child's verbal tutoring concerns the frustrations that he may feel, because the collection of his past experiences being rather limited due to his young age, it is quite likely that he would not have had prior opportunities to analyze much and learn how to behave regarding every type of frustrating situations that he may be confronted with as he grows up.

A clear distinction must be made here between real frustrations and "pseudo frustrations", that is, the subtle attempts the child will make from time to time to test "where the limits are" that he or she is not allowed to overstep with one or other, of both, of his parents.

In the case of a real frustration, it is quite possible that no connection has been made between this particular new annoying nonverbal experience and the collection of impressions that he has already associated in his mind on the verbal level.

The only really efficient way for the child to make these connections is to successfully verbally explain his problem. This will of course be difficult at first for him, since no connection having previously been made verbally between his feelings and this particular type of event, it will not be easy for him to find the proper words to explain why he feels sad and frustrated.

It is, therefore, necessary to question him, discuss the matter with him, explain the various aspects of the problem in view of his answers. We must reason with him (not reason him), and exchange ideas in order for him to acquire the proper vocabulary that will allow him to more easily make the necessary connections at the verbal level. If his emotion is justified, he must be told as much so he does not becomes confused, and the parent must address the causes that are out of the child's control to resolve if possible. 
The more thoroughly these experiences are verbally explored with a child, the denser the network of coherently structured interconnections of his brain will become, and the more he will become able to successfully deal by himself with new situations similar to instances already explored.

Paradoxically, despite the displeasing nature of frustrating experiences, they probably are the most powerful tool at the disposal of parents and educators, because their exploration allows teaching childen to become skilled at working out real social interaction problems.

\section{The Inner Structure of the Human Neocortex}

Now that we have seen how children take their first steps in understanding the world with which they interact, and before examining the manner in which they must be guided to acquire a perception as correct as possible of the world and learn to clearly distinguish between illusion and reality, we will give a closer look to the physical support of the subjective personal model of reality that every child is in the process of constructing in his verbal areas.

We will not be exploring here what we call "consciousness", or "awareness", or "soul", or whatever name may be given to what makes us self aware. In this regard, Paul Chauchard's book "Physiologie de la

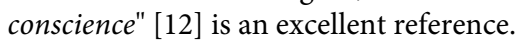

This internal model of reality that each individual builds as he grows up cannot possibly be otherwise than subjective, because it is built uniquely from the person's own perceptions, which does not prevent us from often seeing it as if it was objective reality proper. The clear distinction that must be made between each of our individual personal opinions and what constitutes objective reality is extensively dealt with in reference ([7], Section II).

As previously mentioned, the neocortex is an extremely complex structure that still has not been fully explored and understood. We will therefore comfortably remain at the level of general functional description. The human neocortex is made of approximately 14 billion neurons organized in 6 rather regular layers in the following manner:

One input layer, composed of receptor neurons. It is comprised of separate localized groups of neurons through which the impressions of the senses are received by the neocortex, as well as groups through which our emotions come to our attention, originating in the hypothalamus. The remainder of this input layer receives signals originating from the neocortex itself, which incorporates innumerable backwards synaptic links coming from deeper intercalary layers or even the output layer, which allows already processed signals sets (automatically perceive coherences) to be reintroduced at the input layer ([4], p. 88).

Four intercalary layers, within which it can be assumed that memories are stored, and within which the automatic correlating processes occurs.

One output layer, which includes groups of psychomotor neurons, through which the "orders" of the neocortex are transmitted to other parts of the brain, and to parts of the body and where the coherently resolved perceptions from our senses, and conclusions of our "reasoning" are made available for our conscious mind to become aware of.

This multilayer network is the seat of human conceptual thinking. As already mentioned and strongly emphasized by Chauchard, the processing power of multilayer neural networks is not directly related to the number of neurons that they are made of, but to the number of synaptic interconnections that exist between these neurons $[4,9]$.

Considering that our galaxy contain about 40 billion active stars, we would have to count 2,500 such galaxies to obtain a number of stars that would match the approximate number of synaptic connections contained within this external envelope of the human brain.

Or else, if we consider that 100 million atoms must be aligned to obtain a length of 1 centimeter, it would be necessary to align atoms for a length of 10 kilometers to obtain an equivalent number of atoms. In comparison, let us mention that the most complex artificial neural network ever constructed as of 1998 contained about 1 million neurons linked by barely a few million interconnections.

\section{Automatic Initial Coherence Perception and Resubmission}

At the functional level and in a simplified manner, it could be considered that each time a set of signals is provided in input to as many neurons of the entry layer of the neocortex, the intercalary layers behave as if they were answering the simple following question: "Are there similarities between elements in this set of signals?"

In the visual area for example, similar intensity of neighboring signals on a variety of criteria obviously allows pattern identification in the input signals set, allowing coherent patterns to be automatically selected and provided to the output layer.

Such patterns seem to also be automatically re-fed to the entry layer elsewhere in the non-verbal areas of the neocortex allowing comparison of this new pattern with patterns previously stored, leading to eventual "recognition" of similarity with previous patterns and association to this new pattern of the "reactions" that were previously associated to these stored patterns.

With regard to verbal identification of such patterns or events, given that specific descriptive word sequences, or single words, can be associated to a wide variety of intensities of patterns or events depending on context, many "first glance" coherent description may be automatically conjured up to describe any new, or reconsidered pattern or event.

Given that by structure, only one possible verbal "description" can be fed at a time to the output layer, many "sweeps", so to speak, generally need to be voluntarily made, if the individual wishes to correctly identify the optimal "description" of this particular non verbal pattern or event.

Chauchard's research reveals that in simple neural networks, like the reflex networks in inferior animals, the arrival of sensory messages at the input layer results in the direct activation of specific motor neurons. But in the case of the neocortex, the multitude of receptors of the entry layer, which receive multiple messages non stop from the senses and from the hypothalamus, are not directly connected to the motor neurons of the output layer, and instead permanently activate the complex intercalary network in its entirety ([4], p. 84-91).

These messages will freely circulate inside the neocortex in innumerable synaptic circuits, without necessarily be directed to motor neurons. Such direct activation of motor neurons will then occur only in certain "emergencies" such as cases of burn or needle pricks, and this, only after the appropriate reaction has been learned. Otherwise, the psychomotor neurons will be activated only if we want it.

Chauchard concluded that once activated, the neocortex no longer 
needs to be constantly supplied with sensory messages, to continue operating. It can continue to function autonomously because it can automatically feed itself with stimulations which are the result of the coherent thinking process, on account of its internal structure comprised of feedback links. Every conclusion that we draw from our reasonings is automatically re-fed in input in this manner, and is integrated in a coherent manner into the neurolinguistic structure in place, and thus remains at our disposal like any other information that was integrated from our sensory perceptions and emotions.

A decrease in the number of sensory messages coming from the senses which drives animals to sleep, allows human beings, if they so desire, to become engrossed in their thoughts instead of slipping into sleep.

We can also, at will, and without being driven by any incoming sensory message, activate our motor neurons to execute actions that are not reflex. The very complexity of the intercalary network, in conjunction with the presence of the feedback links therefore allows us not to be the slaves of reflex behavior, nor to the arrival of constant sensory messages.

There exists many types of neurons, but generally speaking, it can be considered that receptor neurons are of the "granular" type while motor neurons, or transmitters, are of the "pyramidal" type. It can also be considered that within the neocortex, granular layers alternate with pyramidal layers.

Motor neurons of a given layer can therefore connect part of their output synapses to the inputs of one of the granular layers which precedes its own layer, and do not necessarily connect all their synapses to the inputs of the following layer of neurons. This is what explains why a sensory impression at the first input layer does not automatically result in a reflex action by means of the last motor neurons layer.

This also explains why newborn babies are so powerless with regard to their environment. Practically no behavior is innate concerning the reactions that they should have in relation to the sensory impressions, except for some basic genetically programmed reflexes like the eyelid closure reflex. They must learn how to react to absolutely everything.

\section{Requestioning}

As mentioned earlier, the problem caused by the fact that many possible "verbal descriptions" are "first glance" candidates to describe any given non verbal event or coherence, is easily circumvented by voluntarily resubmitting to the entry layer the coherence to be described. It is precisely the existence of the backward synaptic links that are an integral part of the network that allows it.This resubmission process even tends to become automatic once the verbal resubmission pattern has been voluntarily established, given the physical presence of the then strengthened backward resubmission paths.

But because we think consciously, each time one of our conclusions seems to make better sense with respect to other related conclusions previously drawn and integrated, we tend to become certain that this new conclusion is the best possible, which tends to irrevocably block the resubmission mechanism for this particular conclusion.

Consequently, instead of naturally being re-questioned when new information could possibly justify reassessment, this conclusion will tend to be integrated as is into the individual's subjective neurolinguistic model of reality, whatever its degree of proximity with respect to objective reality. As a matter of fact, large segments of each our personal models can thus drift little by little away from proper assessment until they provide an individual with a rather warped representation of some aspects of reality.

For the "resubmission mechanism" to operate optimally, it is therefore mandatory that we become aware of this perfectly natural, but constantly threatening inhibiting tendency. This means that if we really want to eventually reach the really appropriate conclusions that should be drawn to completely connect with reality, it is important that we systematically requestion our conclusions, however certain we might momentarily have become of their validity. This is the price to pay for insuring that our eventual final conclusions will in each case be as close as possible to reality.

This is the reason why methods of reflection that involve doubt and requestioning have been so beneficial for Humanity in the past. Let us only think of the outstanding discoveries made by many well known skeptics of the past, such as Socrates, Platon, Descartes, Newton, Einstein, etc. [1]. As we just saw, our brain is biologically and functionally structured to operate optimally when we think in this manner.

But everyone is obviously free to do so, or not, since we have full control over the process. We always remain free to revisualize, or not, our past activities through use of our memories and imagination, to eventually revise, or not, and correct, or not, possible misconceptions or wrong conclusions that we might have accepted as appropriate in the past.

We can also elaborate new concepts by drawing conclusions from the coherences that we have perceived, and consider the effect of these new conclusions on the whole set of conclusions that we have previously drawn, as we consciously re-feed these new conclusions at the entrance layer. This process, that we can repeat at will, is entirely under our control. We have it at our disposal to use to our best advantage, if we so wish.

But it is rather difficult to talk about a concept as abstract as verbal identification of non-verbal events or of abstract concepts, without using visualization. Consequently, to more easily come to grips with this very abstract issue, let us associate a few symbolic images to these concepts, which will render them more substantial.

Let us represent all of the data elements (known and unknown) pertaining to a non-verbal event, by an ordinary white sheet of paper, each atom in the sheet symbolizing a specific characteristic. If we imagine this sheet as being only one atom thick and stretching to infinity in all directions of the plane, we can now visualize an infinite number of atoms corresponding the potentially infinite number of characteristics that any really occurring event may have. This infinite sheet is represented by the large circle in Figure 2.

Given that we never need to have perceived the totality of this infinite number of characteristics of an object, event, concept or emotion before we can objectively understand its nature, we know that for any object, event, concept or emotion that we may think about, there exists a restricted set of characteristics that allows objective understanding of its nature [3,23]. The inner circle in Figure 2 represents this restricted set of objective characteristics that allows objective description and understanding of the object, event, concept or emotion under consideration.

This perfect inner circle consequently represents the complete collection of verifiably valid elements, excluding all others, that should have been considered from the start for us to immediately reach the actual optimal conclusion that would really match reality. 
Citation: Michaud A (2017) On the Relation between the Comprehension Ability and the Neocortex Verbal Areas. J Biom Biostat 8: 331. doi:10.4172/2155-6180.1000331

Page 12 of 15

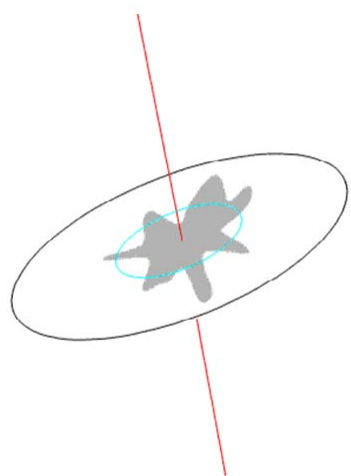

Figure 2: Symbolic representation of verbal identification of non-verbal events or abstract concepts.

Let us be aware at this point, that before any conclusion can be established as perfectly matching reality, as the end result of a retrospective analysis would allow to clearly confirm, it was impossible for us to know in advance which, among all of the elements that we were considering, were really required to obtain it. Similarly some important elements that should have been considered might not have been included, due to any number of reasons, including never having been informed about them.

So, as we reflect on any given issue, following various logical threads, groping for a valid conclusion, from analysis of the dissociated set of elements that appears pertinent at first glance, it is practically impossible that we would not dig a little too deep in some directions, and not deep enough in some others, thus incorporating in the finally retained set some elements that should have remained out of the circle, and not incorporating some others that should have been retained.

Consequently, the set of elements that was initially retained before retrospective reassessment, which led to the first conclusion that was drawn in any given direction, cannot be represented as being enclosed with certainty within such an ideal circle. With respect to this perfect inner circle, this initial set of "assumed" valid elements must instead be symbolized as being circumscribed within the irregular gray shape of Figure 2.

This shape symbolizes that, while in the process of examining whatever concept for the first time: object, idea, memory, impression, complex circumstance, etc., we always are momentarily figuratively "fishing in the dark", poking a little too far here, not far enough there, until enough data has been gathered for an apparently realistic global coherence to appear in the set, but a coherence whose validity cannot, and must not, be assumed prior to retrospective validation.

The fact is that we often retain and integrate into our personal model of reality such presumably imperfect conclusions, without any further questioning, because we like coherence above all, and even an "appearance" of coherence tends to satisfy us.

The infinite line perpendicularly piercing the center of both circles represents our brain's correlating ability. It is represented perpendicular to the white sheet to symbolize that any of even the farthest atoms on the infinite white sheet are still in its "direct line of sight", however far they may be, and could potentially be considered as possibly belonging to the inner circle, also symbolically meaning that nothing is out of reach of our understanding ability.

Figure 3 symbolically represents an arbitrarily large set of our first verbal conclusions that our analyses led us to reach and accept as valid at face value about any issues, prior to retrospective validation.

To visually represent the state of these conclusions with respect to what they should ideally be after the cycle of validationsreconsiderations has been executed sufficiently often to insure objective conclusion [3], let us now associate them to the perfect circles that symbolize the restricted optimal set of elements that should have been considered (Figure 4).

It now become obvious now that some elements of the set initially retained might well lie outside the circle representing the ideal objective set, a state that will become obvious only upon subsequent validation of each element retained and subsequent reconsideration of the set, and that some potentially important elements might well not have been considered at all.

To succeed in readjusting the complete collection of our conclusions, and thus make them fit objective reality more closely, all we need to do is to overcome any not altogether rational impression of certainty that we may harbor about any of them.

The entirely natural re-questioning process with which we are graced and that we tend to unknowingly hinder by becoming certain of some conclusions drawn from first impressions, or insufficient reconsideration, and most importantly, by not confirming the validity of all elements in the set considered, will automatically grind back into action again, and our model of reality will progressively evolve towards matching more and more closely the state which is symbolically represented by Figure 5 .

\section{Taking Control of the Resubmission Process}

As mentioned previously, coherences and conclusions are automatically provided to the output layer of the neocortex from initial

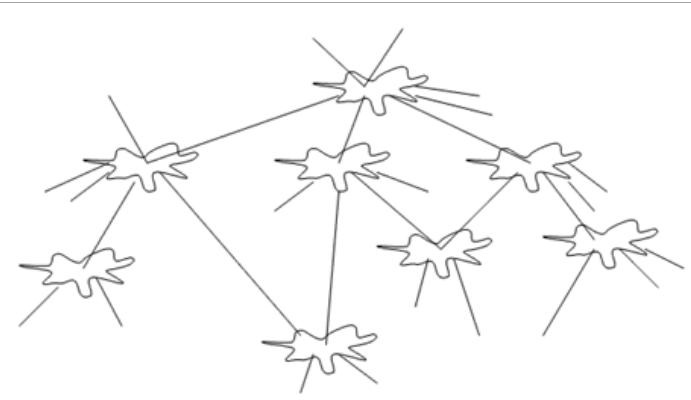

Figure 3: Representation of first coherent verbal conclusions about any issue.

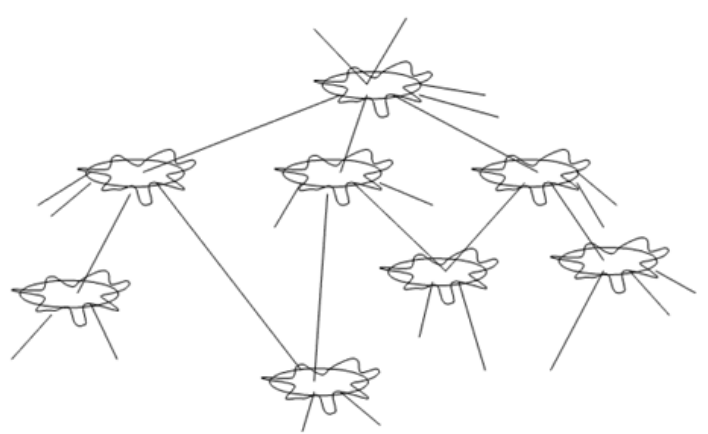

Figure 4: Representation of initial conclusions, versus final objective verbal conclusions 


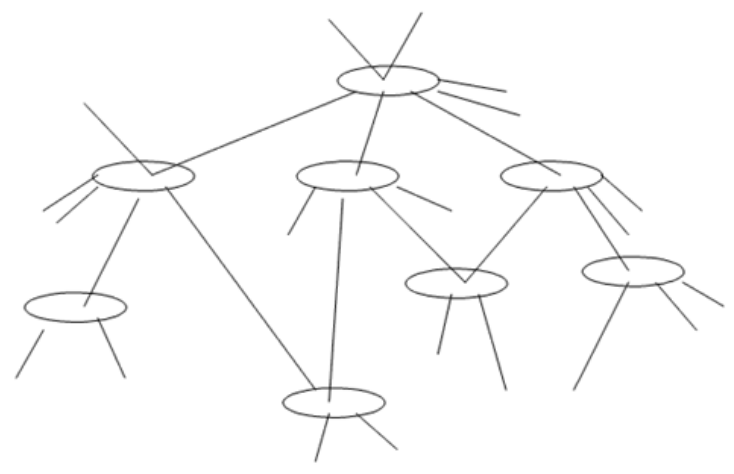

Figure 5: Representation of final objective verbal conclusions.

processing of any entry signals set. These entry signals sets could be made entirely of first level electrochemical signals such as in the visual area, up to and including mixed sets of highly abstract concepts being compared in the verbal areas.

The resubmission process of perceived coherences seems to be totally automatic and out of our control in the centers for images processing, apparently involving only the automatic resubmission sequences required for usable "image imprints" to be stored in the network, where they become available for use by our "conscious mind".

This process seems to be entirely automatic also to a certain extent in the verbal areas, but as we have seen, the process can be brought to a halt when the individual becomes certain of a given conclusion. It is however possible and relatively easy to take control of the verbal resubmission process, as previously discussed.

The traditional linear reasoning mode, which is a training that gives us access to the intellectual rigor essential to all progress ([23], Chapter "Einstein's Playroom"), requires only that all premises, from which any logical reasoning is carried out, be previously verified as being true, which is meant to guarantee that the ensuing conclusion will also be considered true.

However, the logic that must be used to correlate elements in the sets that are fed into the entry layer of verbal areas ([23], Chapter "Defining the Comprehension Process"), requires in addition, that the reference frame, within which each set is considered, be clearly defined in light of the goal being pursued, and re-examined and re-defined if need be, at each stage of the correlation process that will lead to the final conclusion.

The whole collection of links that interconnects the various aspects of our recollections in the neocortex, under the pressure of the use of the words that allow us to think about them, and that we could consider as a hierarchical indexation infrastructure associative by inclusion ([13], Chapter "Hierarchical indexation infrastructure associative by inclusion"), gives us access to all elements of the subjective model of reality ([7], Section II) that each of us has constructed since childhood. In this structure of links, each aspect of the ideas that have been understood activates a substructure of synaptic links that can bring to the forefront of our attention the whole collection of elements of the recollections that we have associated to this aspect of the idea.

Each of these sub-structures are themselves structures of links that associate by inclusion all of the elements to which one of its links is connected. This apparently is the only way that data can be indexed in a multilayer neural network. The number of levels that such an indexation structure can have is unlimited due to the fact that the output layer of the neocortex possesses feedback links towards the preceding layers.

When our attention is drawn to an idea, if an unresolved aspect (Ref: Figure 3) of this idea sufficiently attracts our attention to induce us to question the unresolved issue, we then ask ourselves questions that trigger correlation processes ([23], Chapter "The Correlating Process"). The outcome of such processes is always the eventual perception of new coherences, or "answers", that give us a better understanding of the previously vague aspect that had attracted our attention (Figures 4 and 5).

The correlating process consists in a cascade of successive associations that will gradually "bring" to the forefront of our attention, the set of pertinent correlated elements (of course, only those that the individual will have become aware of), and when a satisfying conclusion has been perceived by the individual, the set of consequently strengthened synaptic interconnections that associate these elements, will naturally become a new hierarchical sub-structure in which each of the newly strengthened links leading to each element of the newly correlated set will be included.

Afterward, the previously unresolved aspect of the idea that caused a question to be formulated, instead of activating the question, will directly activate the set of strengthened synaptic connections leading to the elements whose correlation provided the answer to the question.

On interesting consequence of questioning ourselves about any issue is that once a question has been mentally formulated, a correlating process is always triggered. Once triggered, the process will remain active in the neocortex even if we stop paying attention to this particular issue, discretely keeping on the look out in the background, so to speak, for elements to correlate that may contribute to answer the question ([23], Chapter "Initiating a correlating process").

It seems also that any number of such automatic correlation processes can be initiated by as many clearly formulated questions and that they will remain active at the subconscious level in wait of elements to correlate. They may remain dormant for long periods in wait of missing elements, the perception of which may depend on the outcome other unresolved correlations or on the acquisition of knowledge not yet collected by the individual, or failing such perception or acquisition, may never be resolved.

This automatic feature is at the origin of our intuitions, which we perceive as unexpected sudden flashes of "understanding" on issues that we had been wondering about at some point in the past. The triggering question may have started the process long before the actual resulting correlation is provided at the conscious level, which can occur only after the set of progressively assembled pertaining elements becomes sufficient for a related correlation to be automatically perceived by the neural network, resulting in this sudden "understanding event".

Actually, the only difference between intuition and reasoning is that a conclusion arrived at by intuition is the end result of an automatic subconscious correlation sequence while a conclusion arrived at by reasoning is the end result of a voluntarily guided correlation sequence. In both cases, however, subsequent confirmation of validity of the correlated elements is required to insure the value of the conclusion.

It can be surmised that the synaptic imprints corresponding the elements being considered are not physically displaced in the neocortex, but that temporary connections are rather established to the locations where they are physically stored. These connections seem to become stronger and more durable only for elements belonging to the coherence that constitutes the answer to the question that triggered the process. 
We will now examine a reflection method natural for everyone, because it takes into account a fundamental characteristic of the functioning of multilayer neural networks, that is, the automatic perception of coherences, whatever they may be, in any set of elements, if such coherences exist.

\section{Reasoning by Perception of Coherences}

Logical reasoning involves a process by which one thought regarding the elements of a set leads to a generalizing thought applicable to all cases having the same properties as the elements of the initial set or of a subset of the initial set.

The starting point of any logical reasoning always is a coherence perceived in a set of elements being considered. The reasoning method by perception of coherences allows an undetermined number of elements, or premises, to be included when selecting a set of elements to be analyzed.

This approach was extrapolated from the structured set analysis methods developed by Jean-Dominique Warnier [24-26] and Edsger W. Dijkstra [27], because their approaches perfectly harmonize with Hebb's discoveries regarding the functioning of multilayer neural networks [10].

The development of each logical sequence needs to rigorously follow the criteria of formal logic ([23], Chapter "Einstein's Playroom"), because its successful unfolding mandatorily requires a reference to both mental images and verbal descriptions at every step of the development, which causes the neural network to work with maximum efficiency in both cerebral hemispheres, a technique that can be mastered by learning Euclid's geometric theorems, for example.

Definition of a coherence perceived by correlation:

Coherence criterion: Criterion that appears common to some elements in a set and that allows extraction of these elements to form a subset that will be devoid, by definition, of any exception to this criterion.

Coherences are practically always perceived as a consequence of a question being clearly mentally formulated. To obtain a satisfying result, however, this question must be formulated only after clear awareness of the reference frame within which it is meant to apply has been attained.

The voluntary verbal establishment of such reference frames parallels and enhances the neural network automatic coherence determining search of similarities between data input to the entry layer.

Definition of the reference frame of a set to be considered:

Reference frame: Set of all characteristics that are common to all elements in a set to be considered.

In context, the reference frame that determines what elements will belong to a set can be established in one of two ways:

- Selection of any number of criteria and subsequent search for elements that answer all of these criteria.

- Consideration of a set of elements and identification of all criteria that are common to any given subset of these elements.

The initial sets chosen in search of any conclusion, corresponding to Figure 3, can always be made to evolve towards the ultimate most restricted set of objective elements, corresponding to Figure 5, that allows objective understanding of the concept being explored. This is accomplished by means of resubmitting the initial and subsequent subsets to as many iterations as will be required to identify this most restricted objective set, from which objective conclusions can be drawn.

A detailed example of such a reasoning sequence is described in reference [28]. In fact, this particular example led to the definition of a new space geometry $[29,30]$, which, if confirmed as appropriate, will also confirm the soundness of this reasoning method.

\section{Conclusion}

Objective reality is what is really occurring and whose innumerable signals are detected and provided to the entry layer of our neocortex and whose coherent interpretations are provided to our awareness at its output layer. This means that we are physiologically unable to directly observe this objective reality.

What each individual observes, is rather some sort of personal subjective "model of reality", which is an assembly of all the coherences that he has perceived, and that he keeps on perceiving, in the collection of memories that he has gathered since birth.

In fact, absolutely everything we think we know, everything we believe we have successfully understood, everything that other human beings have communicated to us, everything that we read, and everything that we feel, constitutes the raw information that we have no choice but to work with. We simply have nothing else at our disposal to understand reality.

We systematically name all aspects of this raw information, and endlessly verbally rummage in this heap of prima facie unconnected pieces of information, searching for the various pieces of the Big Puzzle. Every time we think that we have found pieces that seem to fit together, we assemble them. Little by little, an increasingly coherent image of what is occurring in the outside world takes shape, that constitutes our personal model of reality. Coherence is in fact our only reference, or should we say rather, the only guide of our neocortex.

Objective reality is really occurring events of all natures, space, the "present moment", the stable particles making up matter, energy, and all that can be constructed with these elements, from a simple hydrogen atom up to the most extraordinary and complex structure that can be identified in the universe, that is, our neocortex, that allows us to think and will eventually allow us to possibly understand everything, if we proceed in the correct manner.

\section{References}

1. Michaud A (2016) Intelligence and Early Mastery of the Reading Skill. J Biom Biostat 7: 327. doi:10.4172/2155-6180.1000327

2. Lothar P (1998) IP Pawlow - Gesammelte Werke - Über die Physiologie und Pathologie der höhere Nerventätigkeit. Ergon Verlag, Germany.

3. Michaud A (2016). Comprehension Process Overview. J Biom Biostat 7: 317 doi:10.4172/2155-6180.1000317.

4. Chauchard $P$ (1960) Le cerveau et la conscience, Les éditions du Seuil, France

5. Chauchard P (1956) Le langage et la pensée, Que sais-je \#698, Presses Universitaires de France.

6. Shepherd G (1994) Neurobiology. ( $3^{\text {rd }}$ ed.), Oxford University Press

7. Michaud A (1999) A Future as an Heirloom, SRP Books. Smashwords. ISBN 978-0-988-05273-4.

8. Eccles JC (1994) Évolution du cerveau et création de la conscience, Flammarion, France.

9. Anderson (1995) An Introduction to Neural Networks. The MIT Press. ISBN 0-262-01144-1. 
Citation: Michaud A (2017) On the Relation between the Comprehension Ability and the Neocortex Verbal Areas. J Biom Biostat 8: 331. doi:10.4172/2155-6180.1000331

Page 15 of 15

10. Donald O (1949) The Organization of Behavior, Wiley, New York.

11. Jeannette L (1990) Untangling Neural Nets. Dr. Dobb's Journal.

12. Chauchard (1963) Physiologie de la conscience, Que sais-je \#333, Presses Universitaires de France.

13. Michaud A (2003) The Neurolinguistic Foundation of Intelligence, SRP Books. Smashwords. ISBN 978-0-988-05271-0.

14. Hamilton CR (1977) Investigations of perceptual and mnemonic lateralization in monkeys. Academic Press, New York.

15. Hamilton CR (1977) An Assessment of Hemispheric Specialization in monkeys. Ann NY Acad Sci 299: 222-232.

16. Goldman PS, Nauta WJH (1977) Columnar distribution of cortico-cortical fibres in the frontal association, limbic and motor cortex of the developing rhesus monkey. Brain Res 122: 393-413.

17. Levy J (1974) Psychological Implications of Bilateral Asymmetry. In: Dimond SJ, Beaumont JG (eds.) Hemisphere Function in the Human Brain, New York.

18. Basser LS (1962) Hemiplegia of Early Onset and the Faculty of Speech with Special Reference to the Effects of Hemispherectomy. Brain 85: 427-60.

19. Kimura D (1962) Functional Asymmetry of the Brain in Dichotic Listening Cortex 3: 167-178.
20. Chauchard P (1958) Le cerveau humain, Que sais-je \#768, Presses Universitaires de France.

21. Lenneberg EH (1967) Biological Foundations of Language, New York, Wiley.

22. Flechsig $P$ (1920) Anatomie des Menschlichen Gehirns und Rückenmarks auf Myelogenetischen Grundlage, Leipzig, Thienne.

23. Michaud A (1997) Einstein's Operating System, SRP Books, Smashwords. ISBN 978-0-988-05270-3.

24. Warnier J (1981) Logical Construction of Systems

25. Warnier J (1971) Les procédures de traitement et leurs données. Éditions d'Organisation.

26. Warnier J (1971) Pratique de l'organisation des données d'un système Éditions d'Organisation.

27. Dijkstra, Edsger W (1972) Structured Programming. Academic Press. ISBN 0-12-200550-3.

28. Michaud A (1999) Theory of Discrete Attractors, SRP Books, Smashwords. ISBN 978-0-988-05272-7.

29. Michaud A (2004) Expanded Maxwellian Geometry of Space. 4th ed., SRP Books, Smashwords. ISBN: 978-0-988-05274-1.

30. Michaud A (2016) Electromagnetic Mechanics of Elementary Particles. Scolar's Press. ISBN 978-3-659-84420-1. 